\title{
In vitro and In vivo Assessment of Suitable Reference Region and Kinetic Modelling for the mGluR1 Radioligand [ $\left.{ }^{11} \mathrm{C}\right]$ ITDM in Mice
}

\author{
Daniele Bertoglio ${ }^{1},{ }^{1}$ Jeroen Verhaeghe, ${ }^{1}$ Špela Korat, ${ }^{1,2}$ Alan Miranda, ${ }^{1}$ \\ Leonie wyffels, ${ }^{1,2}$ Sigrid Stroobants, ${ }^{1,2}$ Ladislav Mrzljak, ${ }^{3}$ Celia Dominguez, ${ }^{3}$ \\ Longbin Liu, ${ }^{3}$ Mette Skinbjerg, ${ }^{3}$ Ignacio Munoz-Sanjuan, ${ }^{3}$ Steven Staelens ${ }^{1}$ \\ ${ }^{1}$ Molecular Imaging Center Antwerp (MICA), Faculty of Medicine and Health Sciences, University of Antwerp, Universiteitsplein 1, Wilrijk, \\ Belgium \\ ${ }^{2}$ Department of Nuclear Medicine, Antwerp University Hospital, Edegem, Belgium \\ ${ }^{3}$ CHDI Management/CHDI Foundation, Los Angeles, CA, USA
}

\begin{abstract}
Purpose: This study aimed at investigating binding specificity, suitability of reference regionbased kinetic modelling, and pharmacokinetics of the metabotropic glutamate receptor 1 (mGluR1) radioligand $\left[{ }^{11} \mathrm{C}\right]$ ITDM in mice.

Procedures: We performed in vivo blocking as well as displacement of $\left[{ }^{11} \mathrm{C}\right]$ ITDM during positron emission tomography (PET) imaging using the specific mGluR1 antagonist YM-202074. Additionally, we assessed in vitro blocking of $\left[{ }^{3} \mathrm{H}\right]$ ITDM at two different doses of YM-202074. As an alternative to reference region models, we validated the use of a noninvasive image-derived input function (IDIF) compared to an arterial input function measured with an invasive arteriovenous (AV) shunt using a population-based curve for radiometabolite correction and characterized the pharmacokinetic modelling of $\left[{ }^{11} \mathrm{C}\right]$ ITDM in the mouse brain. Finally, we also assessed semi-quantitative approaches.

Results: In vivo blocking with YM-202074 resulted in a decreased $\left[{ }^{11} \mathrm{C}\right]$ ITDM binding, ranging from $-35.8 \pm 8.0 \%$ in pons to $-65.8 \pm 3.0 \%$ in thalamus. Displacement was also markedly observed in all tested regions. In addition, in vitro [ $\left.{ }^{3} \mathrm{H}\right] \mathrm{ITDM}$ binding could be blocked in a dosedependent manner. The volume of distribution $\left(V_{\mathrm{T}}\right)$ based on the noninvasive IDIF ( $V_{\mathrm{T}}$ (IDIF) showed excellent agreement with the $V_{\mathrm{T}}$ values based on the metabolite-corrected plasma input function regardless of the metabolite correction $\left(r^{2}>0.943, p<0.0001\right)$. Two-tissue compartmental model (2TCM) was found to be the preferred model and showed optimal agreement with Logan plot $\left(r^{2}>0.960, p<0.0001\right)$. A minimum scan duration of 80 min was required for proper parameter estimation. SUV was not reliable $\left(r^{2}=0.379, p=0.0011\right)$, unlike the SUV ratio to the SUV of the input function, which showed to be a valid approach.

Conclusions: No suitable reference region could be identified for $\left[{ }^{11} \mathrm{C}\right] \mathrm{ITDM}$ as strongly supported by in vivo and in vitro evidence of specific binding in all brain regions. However, by
\end{abstract}

Electronic supplementary material The online version of this article (https:// doi.org/10.1007/s11307-019-01435-1) contains supplementary material, which is available to authorized users.

Correspondence to: Steven Staelens; $e-m a i l$ : steven.staelens@uantwerpen.be 
applying appropriate kinetic models, $\left[{ }^{11} \mathrm{C}\right] \mathrm{ITDM}$ PET imaging represents a promising tool to visualize mGluR1 in the mouse brain.

Key words: Kinetic modelling, mGluR1, $\left[{ }^{11} \mathrm{C}\right]$ ITDM, PET, Reference region, Mouse, Arteriovenous shunt, Preclinical imaging

\section{Introduction}

Glutamate, the major excitatory neurotransmitter in the brain, plays an essential role in a variety of physiological processes. Among several ionotropic and metabotropic receptors, the metabotropic glutamate receptors (mGluRs) are $G$ protein-coupled receptors involved in the modulation of synaptic transmission and neuronal excitability [1]. The mGluRs of the group I are located post-synaptically, and they include the mGluR type 1 (mGluR1) and type 5 (mGluR5).

Both mGluR1 and mGluR5 have been linked to a number of neurological disorders, including epilepsy, stroke, fragile $\mathrm{X}$ syndrome, Huntington's disease, obsessive-compulsive disorder, Alzheimer's disease, Parkinson's disease, and drug addiction [2]. Thus, given the relevance of group I mGluRs for the evaluation of potential therapeutic interventions, there is a growing interest for in vivo monitoring of group I mGluRs in the living brain which can be achieved by means of positron emission tomography (PET) imaging. Although mGluR1 and mGluR5 share a high degree of homology, they are characterized by a distinct cerebral expression pattern, with mGluR5 mainly distributed in the striatum, hippocampus, and cortex, whereas mGluR1 primarily located in the thalamus and in the cerebellum [3, 4].

While a large body of literature on preclinical and clinical mGluR5 PET imaging is available [5], only a limited number of studies in disease models have been reported for mGluR1 PET imaging [6]. In particular, the application of mGluR1 PET imaging in the mouse brain has been extremely limited to date, an important shortcoming given the relevance of mouse models in understanding the pathophysiology of neurological disorders.

Among the radiotracers for mGluR1 described in the literature, N-[4-[6-(isopropylamino)-pyrimidin-4-yl]-1,3thiazol-2-yl]-N-methyl-4-[ $\left.{ }^{11} \mathrm{C}\right]$ methylbenzamide ([ $\left.\left.{ }^{11} \mathrm{C}\right] \mathrm{ITDM}\right)[7]$ is one of the most promising and better characterized radiotracers in the preclinical (rats and rhesus monkeys) settings [7-9]. Notably, Yamasaki and colleagues [8] claimed that the pons is a suitable reference region for noninvasive kinetic modelling of $\left[{ }^{11} \mathrm{C}\right] \mathrm{ITDM}$ in rats. $\left[{ }^{11} \mathrm{C}\right]$ ITDM has also been employed to investigate changes in mGluR1 levels in a mouse model of Huntington's disease using the pons as reference region [8]. However, as in vivo validation has not been performed in mice, it is not clear yet whether the pons is a receptor-free region suitable as reference region. A proper validated reference region is of the utmost importance since erroneous selection of reference region for quantifying PET signal may lead to significant misinterpretation of PET data.

For these reasons, the first aim of the present study was to validate the specific binding of $\left[{ }^{11} \mathrm{C}\right]$ ITDM and to investigate whether a suitable a reference region exists in the mouse brain. To this end, we performed in vitro blocking of $\left[{ }^{3} \mathrm{H}\right] \mathrm{ITDM}$ as well as in vivo blocking and displacement of $\left[{ }^{11} \mathrm{C}\right]$ ITDM with the specific mGluR1 antagonist YM202074 [10]. Since invasive arterial blood sampling in mice presents several challenges and limitations in the perspective of longitudinal studies, the second aim of the study was to investigate whether an image-derived input function (IDIF) could be used as a valid alternative approach for noninvasive quantification of $\left[{ }^{11} \mathrm{C}\right]$ ITDM PET imaging in the eventual absence of a reference region. The third aim was to characterize suitable kinetic models for $\left[{ }^{11} \mathrm{C}\right]$ ITDM quantification and to assess possible semi-quantitative approaches.

\section{Materials and Methods}

\section{Animals}

Adult male C57BL/6J mice from Jackson Laboratories (Bar Harbour, ME, USA) were used in the study. Details of the animals are provided in the Electronic Supplementary Material (ESM).

\section{Radiotracer Synthesis}

$\left[{ }^{11} \mathrm{C}\right]$ ITDM synthesis was performed on an automated synthesis module (Carbosynthon I, Comecer, The Netherlands) based on [7]. Details of the radiosynthesis are provided in the ESM.

\section{PET Acquisition}

Two Siemens Inveon PET/CT scanners (Siemens Preclinical Solution, Knoxville, USA) were used to acquire the dynamic microPET/computed tomography (CT) images. Animal preparation was performed as previously described [11, 12]. Details of the PET acquisition are provided in the ESM.

\section{Image Reconstruction and Processing}

Details of the image reconstruction and processing are provided in the ESM. 


\section{Metabolite Correction}

In order to generate a population-based metabolite correction to account for peripheral radiometabolism, we measured parent fractions in a cohort of WT mice $(n=3$ per time point) at 5, 15, and $30 \mathrm{~min}$ p.i. The procedure was done adapting the previously described methodology [13] to $\left[{ }^{11} \mathrm{C}\right]$ ITDM. Details of the procedure are provided in the ESM.

\section{Kinetic Modelling}

During the baseline and blocking study, regional TACs were fitted by the Logan plot method [14] using the noninvasive IDIF as previously validated [11]. This approach allows comparative studies to be performed in mice, avoiding blood sampling, which is invasive and not feasible for longitudinal studies. To assess the extent of target occupancy reached during the blocking study with YM-202074, we applied the Lassen plot [15] based on the regional changes in $V_{\mathrm{T}}$ (IDIF, Uncorr) following drug pretreatment. Simultaneously, we evaluated whether future kinetic modelling could rely on a reference model. Parametric maps were generated through voxel-based graphical analysis (Logan plot) with the IDIF (Uncorr) as input function.

In absence of a suitable reference region, full kinetic modelling was now performed by fitting the regional TACs by one-tissue compartmental model (1TCM), two-tissue compartmental model (2TCM), and Logan plot in order to estimate the total volume of distribution $V_{\mathrm{T}}$ (AV shunt, Corr) as well as its noninvasive surrogate $V_{\mathrm{T}}$ (IDIF, Uncorr) using the noninvasive IDIF. The blood volume fraction $\left(V_{\mathrm{B}}\right)$ was fixed at $3.6 \%[16]$ for the compartmental models, while, for the Logan plot, the linear phase $\left(t^{*}\right)$ was calculated from the curve fitting with $t^{*}$ ranging 12.5-20 min depending on the brain region.

Details of the model selection, stability of outcome parameters, and assessment of simplified approaches are provided in the ESM.

\section{Brain Tissue Collection}

Animals were euthanized by decapitation while under anaesthesia and brains were snap-frozen in 2-metylbuthane at $-35{ }^{\circ} \mathrm{C}$ for $2 \mathrm{~min}$ and further preserved at $-80{ }^{\circ} \mathrm{C}$ until use. Sagittal sections $(20 \mu \mathrm{m}$ of thickness) were collected starting at $0.96 \mathrm{~mm}$ lateral according to Paxinos and Franklin [17] in triplicate on Superfrost Plus slides (Thermo Fischer Scientific, USA), using a cryostat (Leica, Germany).

\section{$\left[{ }^{3} H\right] I T D M$ Autoradiography}

To further evaluate the suitability of a reference region, we investigated the effect of mGluR1 blockade on in vitro $\left[{ }^{3} \mathrm{H}\right]$ ITDM autoradiography. Details of the procedure are provided in the ESM.

\section{Statistical Analysis}

Repeated measurements two-way ANOVA was applied to compare in vivo PET and in vitro autoradiography values between baseline and blockade in different regions as well as to evaluate differences between $V_{\mathrm{T}}$ values based on $\mathrm{AV}$ shunt and IDIF as input functions. Pearson's correlation tests were used when assessing relationship between two variables. Pearson's correlation tests as well as Bland-Altman plots were applied to compare $V_{\mathrm{T}}$ (AV shunt, Corr) and $V_{\mathrm{T}}$ (IDIF, Uncorr) values determined with $2 \mathrm{TCM}$ and Logan plot. Analyses were performed with GraphPad Prism (v 6.0) statistical software. The data are represented as mean \pm standard deviation (SD), unless specified otherwise. All tests were two-tailed and significance was set at $p<0.05$.

\section{Results}

\section{Assessment of $\left[{ }^{11}\right.$ C]ITDM Specific Binding Demonstrates Lack of Suitable Reference Region}

To confirm the specificity of $\left[{ }^{11} \mathrm{C}\right]$ ITDM for mGluR 1 and determine whether reference region-based kinetic models could be applied in the mouse brain, first we performed in vivo blocking of $\left[{ }^{11} \mathrm{C}\right] \mathrm{ITDM}$ with the mGluR1 antagonist YM-202074. $\left[{ }^{11} \mathrm{C}\right] \mathrm{ITDM}$ parametric $V_{\mathrm{T}}$ (IDIF,Uncorr) maps of baseline and blockade scans are shown in Fig. 1a. SUV TACs during baseline scan displayed the peak of radioactivity around 30 min post-injection, with the highest uptake in cerebellum and thalamus, followed by a slow wash-out (Fig. 1b). Pretretament with YM-202074 $(20 \mathrm{mg} / \mathrm{kg}$, i.v. 2 min prior to $\left[{ }^{11} \mathrm{C}\right] \mathrm{ITDM}$ injection) resulted in an evident decline in all the investigated regions demonstrating a marked blockade of mGluR1. Hence, $V_{\mathrm{T}}$ (IDIF,Uncorr) values during pretreatment with YM-202074 were significantly reduced (thalamus, cerebellum, striatum, and hippocampus: $p<0.0001$; pons: $p<0.01)$ compared to baseline as shown in Fig. 1c. $V_{\mathrm{T}}$ (IDIF,Uncorr) values during baseline and pretreatment with YM-202074 for each region are reported in Table 1. The largest difference in $V_{\mathrm{T}}$ (IDIF, Uncorr) values was found in thalamus (baseline, $7.06 \pm 0.42 \mathrm{ml} / \mathrm{cm}^{3}$; YM$\left.202074,2.41 \pm 0.27 \mathrm{ml} / \mathrm{cm}^{3} ;-65.8 \pm 3.0 \%, p<0.0001\right)$, while the smallest in pons (baseline, $2.66 \pm 0.18 \mathrm{ml} / \mathrm{cm}^{3}$; YM-202074, $1.73 \pm 0.19 \mathrm{ml} / \mathrm{cm}^{3} ;-35.8 \pm 8.0 \%, p<0.01$ ). Additionally, by applying the Lassen graphical analysis, we could estimate that pretreatment resulted in a mGluR1 occupancy by YM-202074 of $79.2 \%$ (Fig. 1d).

To further support these findings, an in vivo displacement study was performed by injecting YM-202074 $(20 \mathrm{mg} / \mathrm{kg}$, i.v.) $30 \mathrm{~min}$ following start of the scan. SUV summed PET images covering 15-30 min and 60-90 min post-radiotracer injection were generated for visual comparison (Fig. 2a). As 

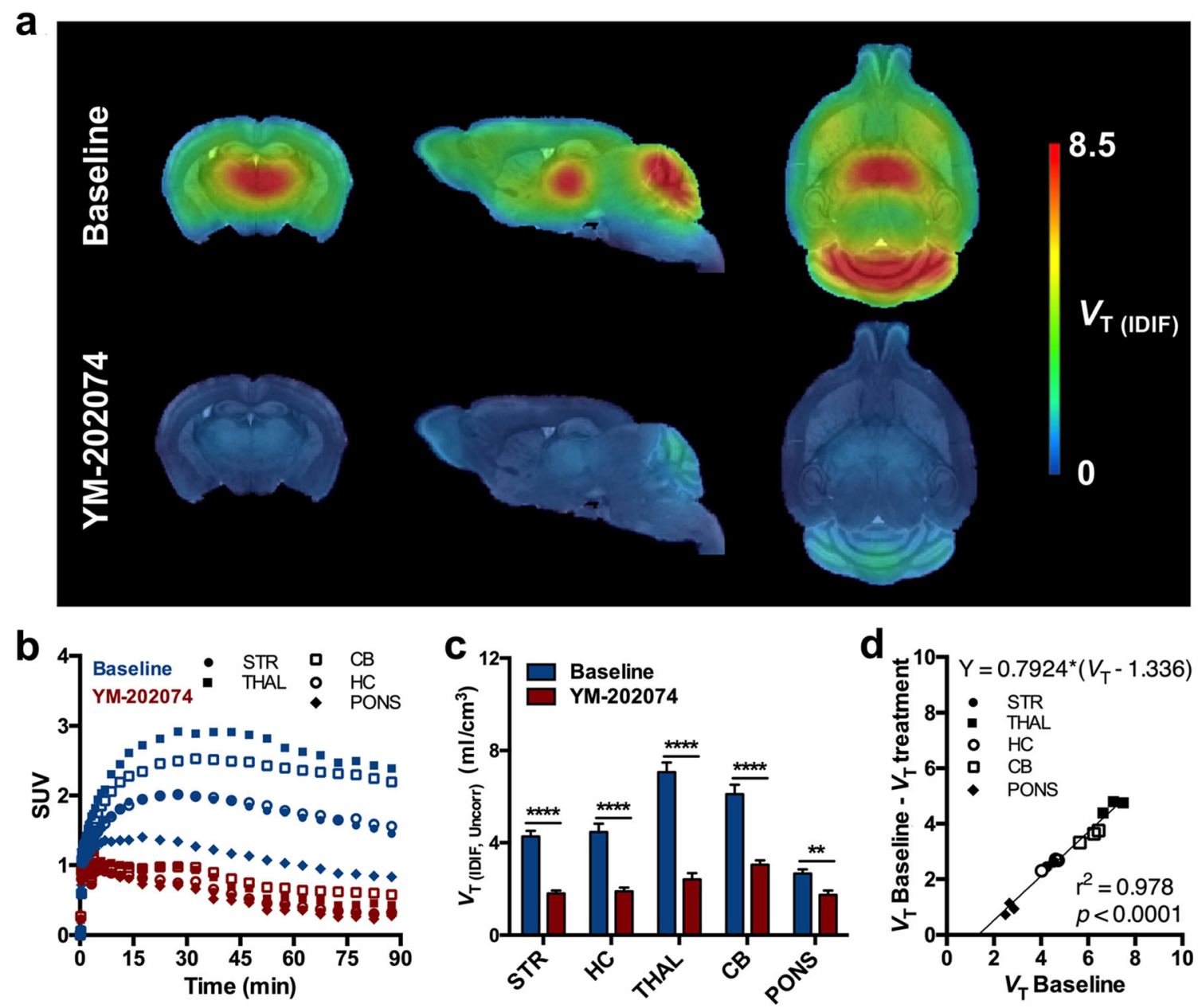

Fig. 1. Effect of in vivo blockade on $\left[{ }^{11} \mathrm{C}\right]$ ITDM binding. a Average parametric maps during baseline and pretreatment with YM202074 (20 mg/kg, i.v.) overlaid onto a MRI mouse brain template for anatomical localization. b SUV time-activity curves and c $V_{\mathrm{T}}$ (IDIF, Uncorr) quantification during baseline and pretreatment with YM-202074. d Lassen graphical analysis estimated a mGluR1 fitted occupancy of $79 \%$. ${ }^{* *} p<0.01,{ }^{* \star \star *} p<0.0001 . n=3 /$ group. STR, striatum; THAL, thalamus; HC, hippocampus; $\mathrm{CB}$, cerebellum.

shown in the SUV TACs (Fig. 2b), $\left[{ }^{11} \mathrm{C}\right]$ ITDM displacement after injection of YM-202074 resulted in a robust and manifest effect in the whole brain.

Finally, we performed an in vitro blocking study with $\left[{ }^{3} \mathrm{H}\right] \mathrm{ITDM}$ autoradiography. As shown in Fig. 3, coincubation of $\left[{ }^{3} \mathrm{H}\right] \mathrm{ITDM}$ with YM-202074 resulted in a

Table 1. Effect of mGluR1 blockade on $\left[{ }^{11} \mathrm{C}\right] \mathrm{ITDM}$ binding. $V_{\mathrm{T}}$ (IDIF, Uncorr) quantification during baseline and pretreatment with YM-202074 determined by Logan plot based on 90 min acquisition

\begin{tabular}{llll}
\hline Region & $\begin{array}{l}\text { Baseline } \\
V_{\mathrm{T}}\left(\mathrm{ml} / \mathrm{cm}^{3}\right) \\
\end{array}$ & \multicolumn{2}{l}{ Pretreatment with YM-202074 } \\
\cline { 3 - 4 } & Mean (SD) & Mean (SD) & Decrease (\%) \\
\hline Striatum & $4.27(0.25)$ & $1.80(0.12)$ & $-57.8^{* * * * *}$ \\
Thalamus & $7.06(0.42)$ & $2.41(0.27)$ & $-65.8^{* * * *}$ \\
Hippocampus & $4.46(0.37)$ & $1.89(0.16)$ & $-57.6^{* * * *}$ \\
Cerebellum & $6.11(0.41)$ & $3.04(0.19)$ & $-50.2^{* * * *}$ \\
Pons & $2.66(0.18)$ & $1.73(0.19)$ & $-34.9^{* *}$ \\
\hline
\end{tabular}

$n=3$

$* * p<0.01, * * * * p<0.0001$ statistically significant decline in a dose-dependent manner in striatum, hippocampus, thalamus, cerebellum ( $p$ $<0.0001)$, and pons $(p<0.05)$.

\section{Image-Derived Input Function Allows Noninvasive $V_{T}$ Quantification}

Since no suitable reference region was present in the mouse brain, we investigated whether an IDIF is accurate to circumvent the need for an invasive input function which disables longitudinal studies.

In order to validate the noninvasive IDIF, $V_{\mathrm{T}}$ (IDIF, Corr) and $V_{\mathrm{T}}$ (IDIF, Uncorr) were estimated and compared to the $V_{\mathrm{T}}$ (2TCM) determined using the radiometabolite-corrected plasma activity input function based on the AV shunt measurement $V_{\mathrm{T}}$ (AV shunt, Corr). Average plasma SUV TACs corrected and uncorrected for radiometabolism are shown in Fig. $4 \mathrm{a}$, with the derived $V_{\mathrm{T}}$ (AV shunt, Corr) and $V_{\mathrm{T}}$ (AV shunt, Uncorr) values displaying high agreement $\left(r=0.964, r^{2}=\right.$ 

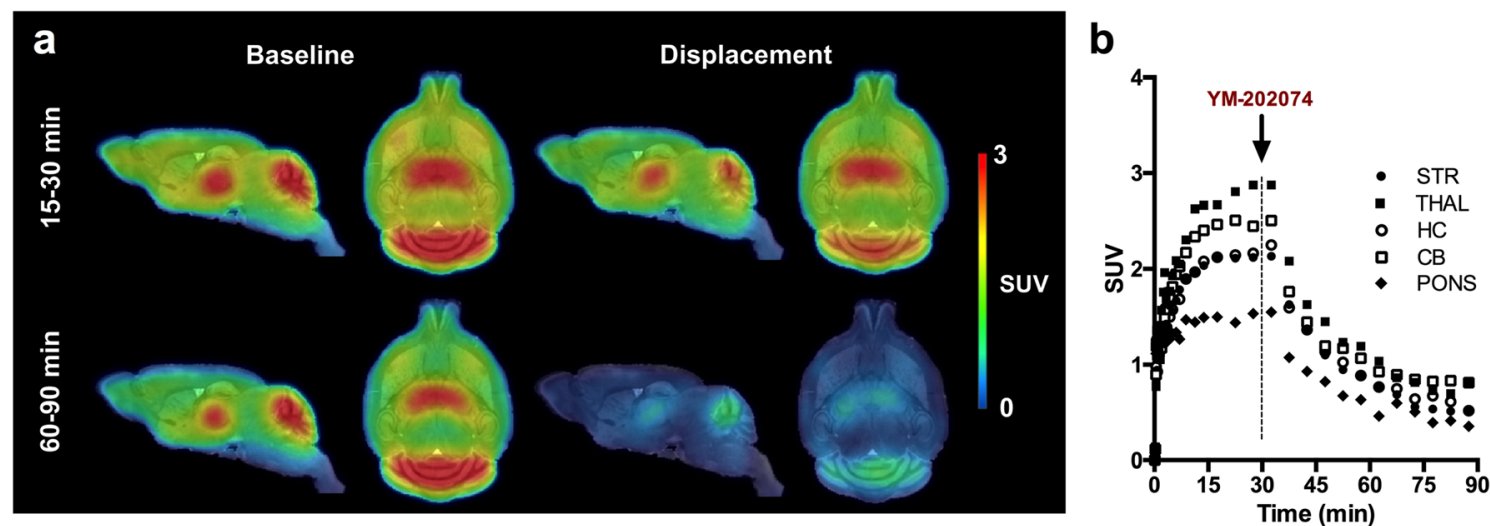

Fig. 2. Displacement of $\left[{ }^{11} \mathrm{C}\right]$ ITDM. a SUV images based on $15-30 \mathrm{~min}$ and $60-90 \mathrm{~min}$ during baseline and displacement with YM-202074 (20 mg/kg, i.v.) injected $30 \mathrm{~min}$ after radiotracer. Images are overlaid onto a MRI mouse brain template for anatomical localization. b SUV time-activity curves indicated $\left[{ }^{11} \mathrm{C}\right]$ ITDM was displaced in all regions. Arrow indicates the injection of YM-202074. $n=3$ /group. STR, striatum; THAL, thalamus; HC, hippocampus; CB, cerebellum.

0.929, $p<0.0001$ ) (Fig. 4b). Comparison of the invasive (AV shunt) and noninvasive (IDIF) input functions revealed the same peak values; however, the IDIF tail values were higher compared to the equivalent invasive values either correcting (Fig. 4c) or not (Fig. 4d) for radiometabolites. Accordingly, noninvasive $V_{\mathrm{T}}$ (IDIF) values corrected ( $V_{\mathrm{T}}$ (IDIF, Corr) $)$ and uncorrected $\left(V_{\mathrm{T}}\right.$ (IDIF, Uncorr) $)$ for plasma metabolism highly correlated with the $V_{\mathrm{T}}$ (AV shunt, Corr) values $\left(r^{2}=\right.$ $0.971, p<0.0001$; and $r^{2}=0.977, p<0.0001$, respectively) with a linear relationship (Fig. 4e, f) indicating that the noninvasive $V_{\mathrm{T}}$ (IDIF) values were proportional to the invasive ones. Noteworthy, the use of the radiometabolitecorrected plasma IDIF did not improve the relation of $V_{\mathrm{T}}$
(IDIF,corr) to $V_{\mathrm{T}}$ (AV shunt, Corr); thus, the uncorrected IDIF could be used as noninvasive input function to determine $V_{\mathrm{T}}$ (IDIF, Uncorr) in future longitudinal studies.

\section{Pharmacokinetic Analysis of $\left[{ }^{11}\right.$ C]ITDM}

Following evidence of the lack of a suitable reference region for $\left[{ }^{11} \mathrm{C}\right] \mathrm{ITDM}$ and the validation of the noninvasive IDIF for $V_{\mathrm{T}}$ (IDIF, Uncorr) quantification, we evaluated several models (Logan plot, 1TCM, and 2TCM) for estimation of the $V_{\mathrm{T}}$ based on invasive radiometabolite-corrected plasma activity $\mathrm{AV}$ shunt $\left(V_{\mathrm{T}}\right.$ ( $\mathrm{AV}$ shunt, Corr) $)$ as well as the

a
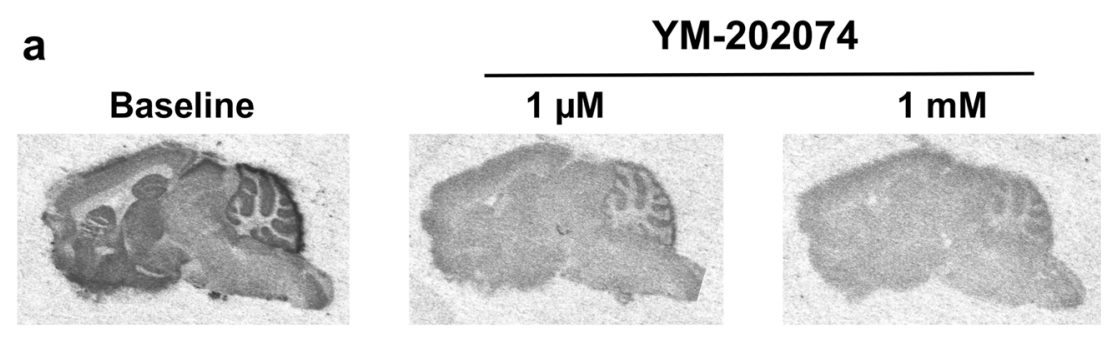

b

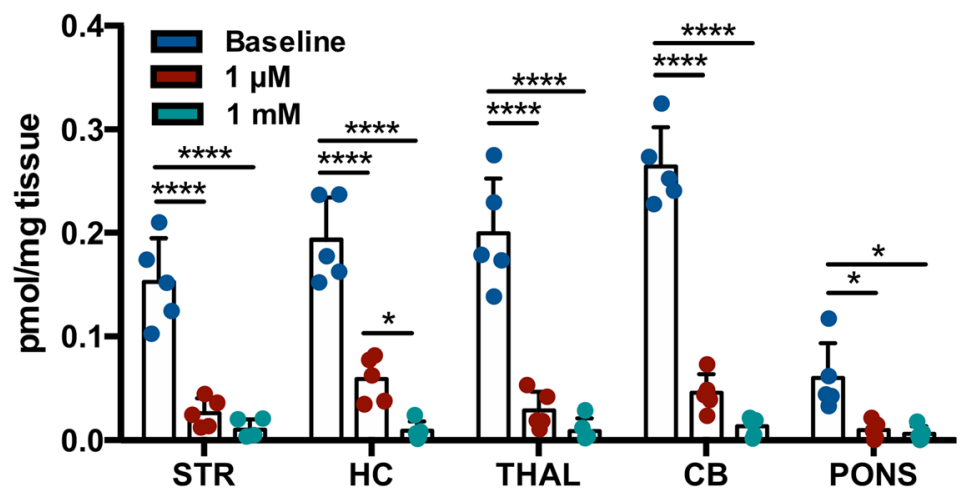

Fig. 3. Effect of in vitro blockade on $\left[{ }^{3} \mathrm{H}\right] \mathrm{ITDM}$ binding. a Representative autoradiograms of $\left[{ }^{3} \mathrm{H}\right] \mathrm{ITDM}$ during baseline and blocking with YM-202074 in the same brain. b Quantification of [ $\left.{ }^{3} \mathrm{H}\right]$ ITDM for baseline and coincubation with YM-202074 $(1 \mu \mathrm{M}$ and $1 \mathrm{mM}$ ). $n=5$ /group. ${ }^{*} p<0.05,{ }^{* * \star *} p<0.0001$. STR, striatum; THAL, thalamus; HC, hippocampus; CB, cerebellum. 

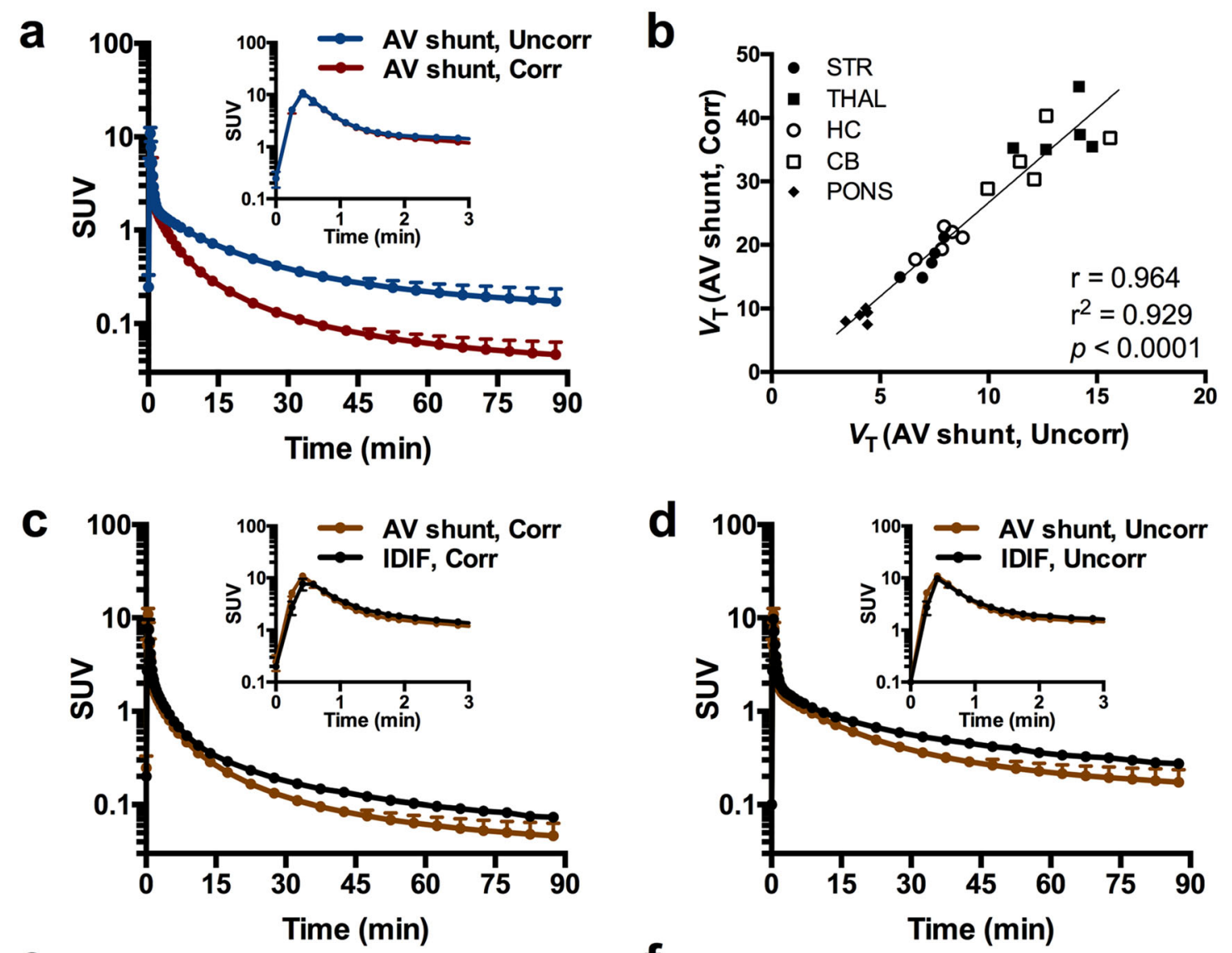

e
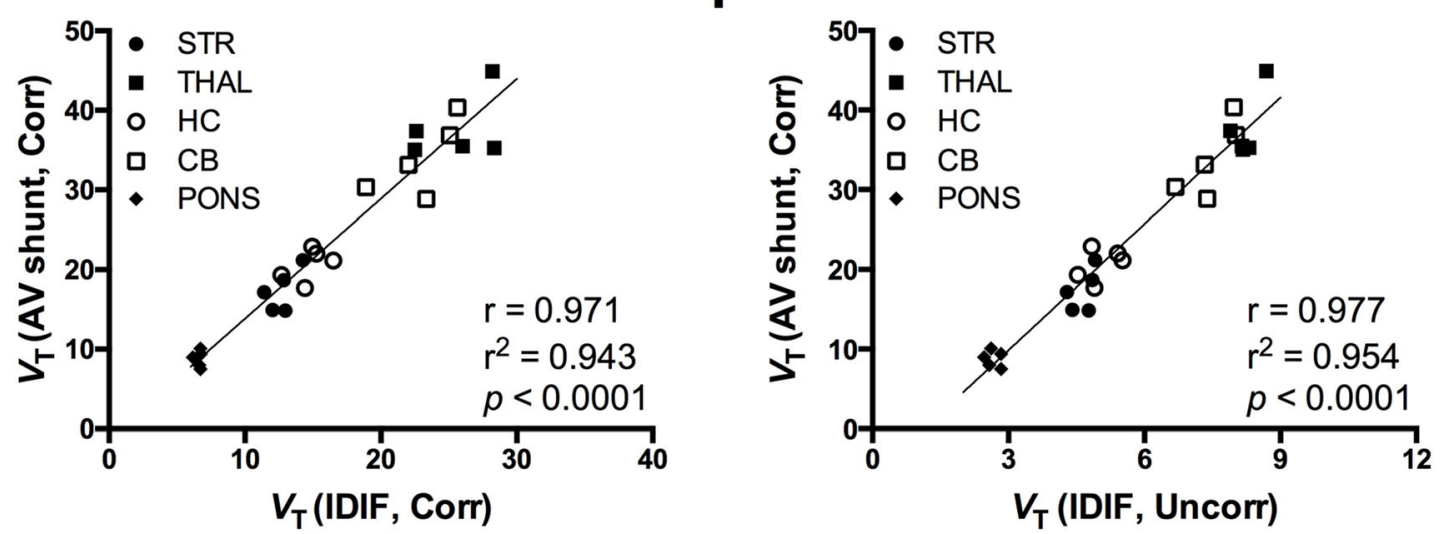

Fig. 4. Comparison of invasive and noninvasive input functions for $\left[{ }^{11} \mathrm{C}\right] \mathrm{ITDM}$ quantification. a Average plasma SUV timeactivity curves (TACs) corrected and uncorrected for radiometabolism and $\mathbf{b}$ correlation between $V_{T}$ (AV shunt) based on 2TCM using plasma input function corrected (AV shunt, Corr) and uncorrected for radiometabolism (AV shunt, Uncorr). c Comparison between average SUV TACs for invasive (AV shunt) and noninvasive (IDIF) input functions corrected and $\mathbf{d}$ uncorrected for plasma radiometabolism. Both $V_{\mathrm{T}}$ (IDIF) values based on e 2TCM corrected (IDIF, Corr) and $\mathbf{f}$ uncorrected (IDIF, Uncorr) for plasma radiometabolites showed excellent correlation with $V_{\mathrm{T}}$ (AV shunt, Corr) based on 2TCM using the metabolite-corrected plasma input function. Solid line represents the linear regression. Data are presented as mean \pm SEM. $n=5$. STR, striatum; THAL, thalamus; HC, hippocampus; $\mathrm{CB}$, cerebellum.

noninvasive $V_{\mathrm{T}}$ (IDIF, Uncorr). According to the AIC (model with the lowest value is the preferred), 2TCM was the desired model (Suppl. Table 1, see ESM), in agreement with the MSC (preferred model has the highest value) (Suppl. Table 2, see ESM). Thus, 1TCM was excluded as it did not fit the data as displayed in the representative fitting in Suppl. Fig. 3 (see ESM).

Both $V_{\mathrm{T}}$ (AV shunt, Corr) and $V_{\mathrm{T}}$ (IDIF, Uncorr) values determined using 2TCM and Logan plot based on $90 \mathrm{~min}$ acquisition resulted in high agreement $\left(V_{\mathrm{T}}\right.$ (AV shunt, Corr): $r^{2}=0.960, p<0.0001 ; V_{\mathrm{T}}$ (IDIF, Uncorr) $: r^{2}=0.986, p$ 
Table 2. $\left[{ }^{11} \mathrm{C}\right] \mathrm{ITDM}$ pharmacokinetics. In vivo kinetic parameters of $\left[{ }^{11} \mathrm{C}\right] \mathrm{ITDM}$ determined using $2 \mathrm{TCM}$ and Logan plot based on 90 min acquisition with metabolite-corrected plasma $\mathrm{AV}$ shunt as input function $\left(V_{\mathrm{T}}\right.$ (AV shunt, Corr)

\begin{tabular}{|c|c|c|c|c|c|c|c|c|}
\hline \multirow[t]{3}{*}{ Region } & \multicolumn{6}{|l|}{$2 \mathrm{TCM}$} & \multirow{2}{*}{\multicolumn{2}{|c|}{$\frac{\text { Logan }}{V_{\mathrm{T}}\left(\mathrm{ml} / \mathrm{cm}^{3}\right)}$}} \\
\hline & \multirow{2}{*}{$\begin{array}{l}K_{1}\left(\mathrm{ml} / \mathrm{cm}^{3} / \mathrm{min}\right) \\
\text { Mean }(\mathrm{SD})\end{array}$} & \multirow{2}{*}{$\begin{array}{l}k_{2}(\text { per min) } \\
\text { Mean }(\mathrm{SD})\end{array}$} & \multirow{2}{*}{$\begin{array}{l}k_{3}(\text { per min) } \\
\text { Mean (SD) }\end{array}$} & \multirow{2}{*}{$\begin{array}{l}k_{4}(\text { per min) } \\
\text { Mean (SD) }\end{array}$} & \multicolumn{2}{|l|}{$V_{\mathrm{T}}\left(\mathrm{ml} / \mathrm{cm}^{3}\right)$} & & \\
\hline & & & & & Mean (SD) & COV (\%) & Mean (SD) & $\operatorname{COV}(\%)$ \\
\hline Striatum & $0.53(0.14)$ & $1.24(0.50)$ & $0.84(0.17)$ & $0.020(0.005)$ & $17.3(2.6)$ & 15.4 & $15.9(2.6)$ & 16.5 \\
\hline Thalamus & $0.73(0.44)$ & $1.02(0.73)$ & $0.91(0.47)$ & $0.016(0.008)$ & $37.6(4.2)$ & 11.1 & $30.0(4.6)$ & 15.4 \\
\hline Hippocampus & $0.49(0.13)$ & $1.26(0.51)$ & $0.81(0.15)$ & $0.017(0.005)$ & $20.6(2.1)$ & 10.1 & $18.9(2.1)$ & 11.1 \\
\hline Cerebellum & $0.67(0.26)$ & $1.33(0.65)$ & $0.95(0.22)$ & $0.012(0.003)$ & 33.9 (4.7) & 13.9 & 31.5 (3.9) & 12.4 \\
\hline Pons & $0.63(0.07)$ & $1.10(0.27)$ & $0.49(0.11)$ & $0.025(0.003)$ & $8.8(1.0)$ & 11.8 & $8.5(0.9)$ & 10.5 \\
\hline
\end{tabular}

$n=5$

$<0.0001)$ without deviating from the identity line as shown in Suppl. Fig. 4 (see ESM). Accordingly, the Bland-Altman plots revealed only a negligible bias for both $V_{\mathrm{T}}$ (AV shunt, Corr) $(-4.97 \%)$ and $V_{\mathrm{T}}$ (IDIF, Uncorr) $(+0.15 \%)$ (Suppl. Fig. 4 , see ESM). The in vivo kinetic parameters of $\left[{ }^{11} \mathrm{C}\right] \mathrm{ITDM}$ based on 2TCM and Logan plot using the metabolitecorrected plasma input function during 90 min acquisition are described in Table 2.

Finally, we investigated the time stability of parameter

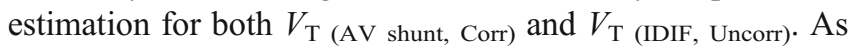
depicted in Fig. 5, shortening the scan duration resulted in a large inter-individual variability of $V_{\mathrm{T}}$ (AV shunt, Corr) values based on both 2TCM and Logan plot, and an underestimation for $V_{\mathrm{T}}$ (IDIF, Uncorr) values. For instance, reducing the acquisition time to $60 \mathrm{~min}$ showed an average bias for cerebellum based on $2 \mathrm{TCM}$ of $-13.1 \pm 13.2 \%$ (Fig. 5a) and $-13.7 \pm 4.8 \%$ (Fig. $5 \mathrm{~b}$ ) for $V_{\mathrm{T}}$ (AV shunt, Corr) and $V_{\mathrm{T}}$ (IDIF, Uncorr), respectively. Similarly, Logan plot resulted in an average bias of $-6.8 \pm 8.1 \%$ (Fig. $5 \mathrm{c}$ ) and $-11.2 \pm 5.1 \%$ (Fig. 5d) for $V_{\mathrm{T}}$ (AV shunt, Corr) and $V_{\mathrm{T}}$ (IDIF, Uncorr), respectively. Thus, an acquisition of $80 \mathrm{~min}$ or longer is required in order to obtain accurate parameter estimates.

\section{Assessment of Simplified Approaches for $\left[{ }^{11}\right.$ C]ITDM Measurement}

Given the long PET acquisition required for parameter estimation, we explored whether a simplified approach for measurement of $\left[{ }^{11} \mathrm{C}\right] \mathrm{ITDM}$ could be applied. Specifically, we compared the calculated $V_{\mathrm{T}}$ values based on radiometabolite-corrected plasma activity ( $V_{\mathrm{T}}$ (AV shunt, Corr) to SUV as well as the SUV ratio of tissue uptake to SUV of input function (both IDIF and AV shunt). The use of SUV did not properly relate to the $V_{\mathrm{T} \text { (Av shumt Corr) }}$ values $\left(r^{2}=0.379\right.$, $p=0.0011$ ) (Fig. 6a). SUVR values based on SUV of the uncorrected IDIF, SUVR (IDIF, Uncorr), correlated well to $V_{\mathrm{T}}$ (AV shunt, Corr) $\left(r^{2}=0.907, p<0.0001\right)$ (Fig. 6b) while the same approach based on SUVR (AV shunt, Corr) values resulted in a less accurate measurement $\left(r^{2}=0.692, p<0.0001\right)$ (Fig. 6c).

\section{Discussion}

The present study described in vivo and in vitro validation of $\left[{ }^{11} \mathrm{C}\right] \mathrm{ITDM}$ specific binding to mGluR1 in the mouse brain, investigated the use of an IDIF for noninvasive kinetic modelling for future longitudinal studies, and characterized pharmacokinetic models for $\left[{ }^{11} \mathrm{C}\right]$ ITDM imaging in mice.

Since, to the best of our knowledge, no in vivo validation of $\left[{ }^{11} \mathrm{C}\right] \mathrm{ITDM}$ binding to mGluR1 has been reported in the mouse brain, the primary aim of this work was to investigate whether a region devoid of specific $\left[{ }^{11} \mathrm{C}\right] \mathrm{ITDM}$ binding existed and thus reference region-based kinetic models could be applied. We demonstrated lack of a suitable reference region in the mouse brain for $\left[{ }^{11} \mathrm{C}\right]$ ITDM. As a first indication, in vivo pretreatment with the highly selective mGluR1 antagonist YM-202074 resulted in a significant blockade in the whole brain, primarily in the mGluR1-rich regions (thalamus and cerebellum), but also in low mGluR1 density regions (pons). This evidence was further supported by the in vivo displacement study, which clearly showed a manifest decline in $\left[{ }^{11} \mathrm{C}\right] \mathrm{ITDM}$ binding following the administration of YM-202074 in the mouse brain, including pons. Based on these findings, we conclude that reference region-based kinetic modelling is not possible for $\left[{ }^{11} \mathrm{C}\right]$ ITDM in mice since no region devoid of specific tracer binding exist [18]. To the best of our knowledge, only one study investigated $\left[{ }^{11} \mathrm{C}\right] \mathrm{ITDM}$ binding changes in the mouse brain, quantifying mGluR1 in a model of Huntington's disease using a reference region-based (pons) kinetic modelling [8]. However, as no in vivo validation was performed, the findings based on such analysis should be interpreted with care. Indeed, if neurological disorders, ageing, or their combination have an effect on $\left[{ }^{11} \mathrm{C}\right] \mathrm{ITDM}$ specific binding in the pons, this could severely affect reference region-based $\left[{ }^{11} \mathrm{C}\right]$ ITDM quantification and lead to significant misinterpretations of the outcome.

Small animal PET imaging features a relative limited resolution for in vivo imaging in relation to the size of the rodent brain; thus, partial volume and spill over effects (PVE) might occur between high contrast regions [19]. Given the anatomical proximity of cerebellum and pons, high and low uptake regions for $\left[{ }^{11} \mathrm{C}\right] \mathrm{ITDM}$ respectively, it 

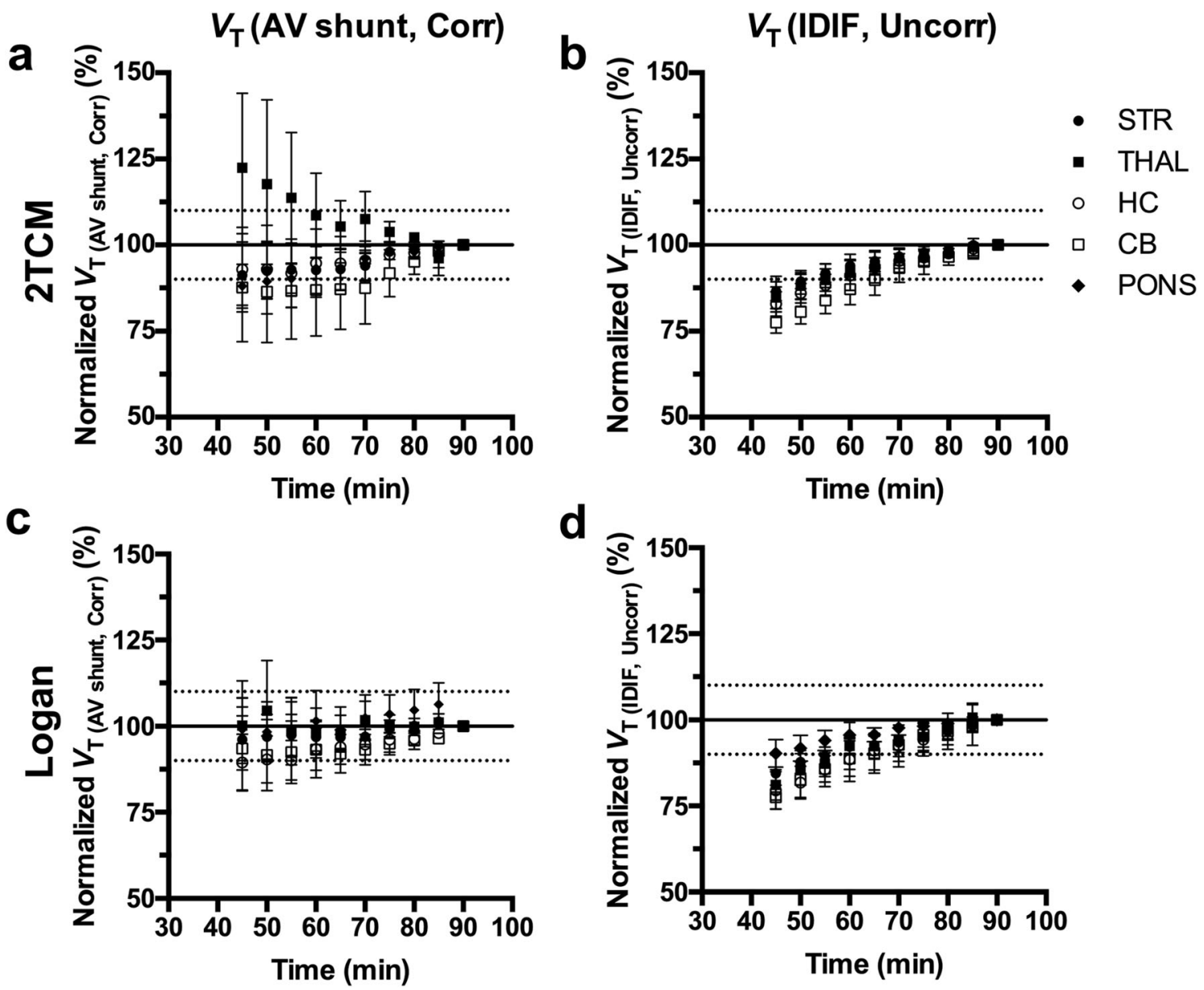

d

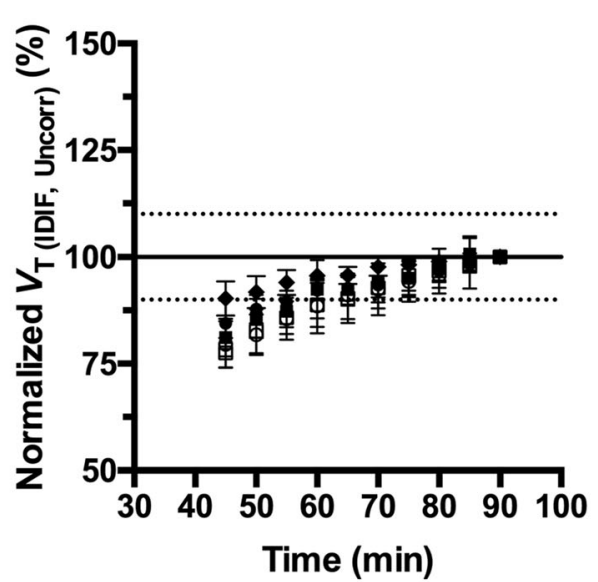

Fig. 5. Time stability of parameter estimation in different brain regions. a $V_{T}$ (AV shunt, Corr) and $\mathbf{b} V_{T}$ (IDIF, Uncorr) calculated using 2TCM and $\mathbf{c}$, d Logan plot normalized to the values obtained during 90 min acquisition. $n=5$. STR, striatum; THAL, thalamus; $\mathrm{HC}$, hippocampus; $\mathrm{CB}$, cerebellum.

cannot be excluded that at least a portion of the changes observed in pons might be related to spill in from the cerebellum. In order to investigate this eventuality, we performed in vitro blockade of $\left[{ }^{3} \mathrm{H}\right] \mathrm{ITDM}$ with different doses of YM-202074, proving blockade of $\left[{ }^{3} \mathrm{H}\right] \mathrm{ITDM}$ specific binding occurred in a dose-dependent manner in all regions, pons included. Therefore, since in vitro validation supported the in vivo findings, we could exclude that the changes observed in the pons were PVE-related.

Noteworthy, when injected in rats, the radiolabelled YM$202074\left(\left[{ }^{11} \mathrm{C}\right] \mathrm{YM}-202074\right)$ in vivo metabolism was fairly rapid [20]. Thus, it is conceivable that the extent of in vivo blockade measured (79\%) could even be underestimated in relation to the total amount of YM-202074 injected. a

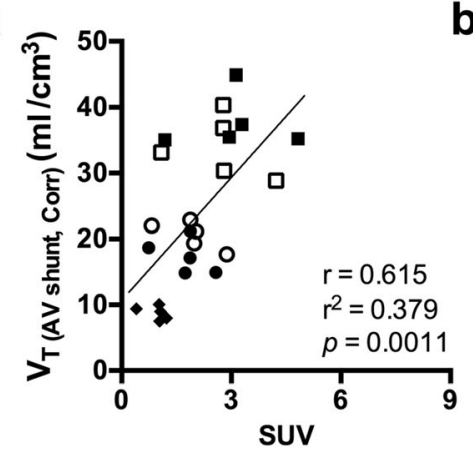

b

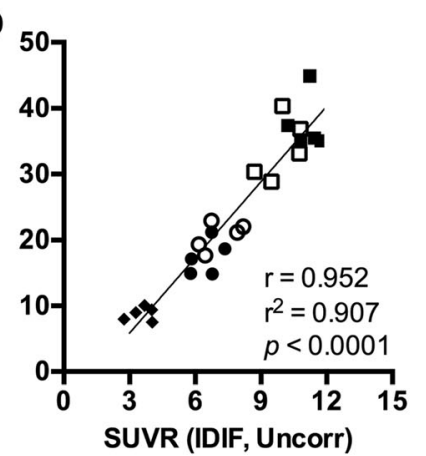

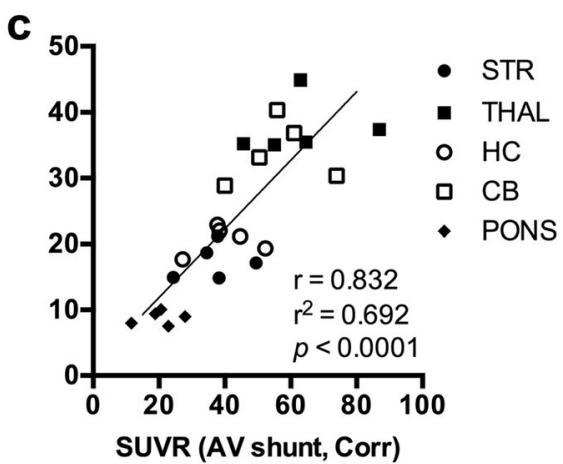

Fig. 6 Assessment of simplified approaches for $\left[{ }^{11} \mathrm{C}\right]$ ITDM measurement. a $V_{\mathrm{T}}$ (AV shunt, Corr) values are plotted in relation to SUV (60-90 min), b SUVR (60-90 min) using as reference the SUV of the input function based on IDIF (IDIF, Uncorr) or c AV shunt (AV shunt, Corr). Dashed line depicts the identity line. $n=5$. STR, striatum; THAL, thalamus; HC, hippocampus; CB, cerebellum; SUVR, SUV of tissue to reference input function. 
Nonetheless, as metabolites of YM-202074 do not apply to an in vitro setting [20], the agreement observed between the in vitro and in vivo blockade studies confirmed the lack of suitable reference region. In order to further investigate the extent of in vivo specific binding in the pons, we are currently testing an equally potent but more stable compound to block $\left[{ }^{11} \mathrm{C}\right] \mathrm{ITDM}$ binding.

The lack of suitable reference region implies the need of measuring an input function in order to perform kinetic modelling of $\left[{ }^{11} \mathrm{C}\right]$ ITDM. However, invasive arterial blood sampling in mice presents several challenges and limitations in the perspective of longitudinal studies due to the small amount of blood collectable and since it is an end of life procedure. Hence, an attractive approach to perform noninvasive quantification is the application of an IDIF [21] to bypass the need for an invasive input function as we previously validated in mice over an atereriovenous shunt for the mGluR5 radiotracer $\left[{ }^{11} \mathrm{C}\right] \mathrm{ABP} 688$ [11]. Similar to the findings for $\left[{ }^{11} \mathrm{C}\right] \mathrm{ABP} 688$ [11], the IDIF overestimated the tail of the $\left[{ }^{11} \mathrm{C}\right] \mathrm{ITDM}$ input function, thus resulted in lower $V_{\mathrm{T}}$ values. However, $V_{\mathrm{T}}$ (IDIF, Uncorr) values showed excellent correlation with the $V_{\mathrm{T}}$ using the metabolitecorrected plasma AV shunt as input function ( $V_{\mathrm{T}}$ (AV shunt, Corr) $\left(r=0.977, r^{2}=0.954, p<0.0001\right)$, supporting the applicability of this noninvasive approach for $\left[{ }^{11} \mathrm{C}\right] \mathrm{ITDM}$ quantification.

Importantly, mGluRs have been described in the rodent heart [22]. In order to exclude that the higher uptake measured with the IDIF compared to the AV shunt was related to specific binding in myocardiocytes, we compared the IDIF of the same animals during baseline and pretreatment as well as displacement with YM-202074. As for both paradigms no difference in the IDIF was observed (Suppl. Fig. 5, see ESM), the higher values in the IDIF are likely to be due to non-displaceable activity spilling-in from the myocardium, which becomes visible with the decline of blood activity. Indeed, although other organs surround the heart, lungs are characterized by extremely low uptake and liver is not in close proximity to the VOI.

While only intact radiotracer has been described in the rat brain, in vivo metabolism of $\left[{ }^{11} \mathrm{C}\right] \mathrm{ITDM}$ has been reported in the plasma of rats and non-human primates [7], with nearly $40 \%$ of intact radiotracer $30 \mathrm{~min}$ p.i. in parallel with the generation of polar metabolites that do not penetrate the blood-brain barrier. Accordingly, we measured around $31 \%$ of intact radioligand at $30 \mathrm{~min}$ p.i. in mice. However, the small blood volume in mice does not allow collection of multiple blood samples for individual metabolite correction with standard techniques. For this reason, we generated a population-based metabolite curve in order to adjust for the peripheral metabolism and to validate the applicability of a noninvasive IDIF over the metabolite-corrected plasma input function. A limitation in the use of a population-based correction is the need of high inter-individual reproducibility; otherwise, under- or overestimations in the quantification may be introduced since the same curve is applied to all the subjects as we recently reported for the radioligand $\left[{ }^{11} \mathrm{C}\right]$ UCB-J [13]. Since correcting the noninvasive IDIF for metabolism did not improve the agreement to $V_{\mathrm{T}}$ (AV shunt, Corr), we will use the uncorrected IDIF as noninvasive input function. Nonetheless, before studying the radioligand in disease-specific animal models, it is recommended to verify whether animals with different genotype or diseased condition are characterized by altered metabolism.

Kinetic analysis was performed by comparing 1TCM, 2TCM, and Logan plot. According to visual assessment and model selection approaches (AIC and MSC), 1TCM was excluded as it did not fit the data, while both $2 \mathrm{TCM}$ and Logan plot proved to be valid alternatives. In addition, time stability of the $V_{\mathrm{T}}$ (AV shunt, Corr) and $V_{\mathrm{T}}$ (IDIF, Uncorr) estimations for both 2TCM and Logan plot were investigated. Shortening the scan duration destabilized $V_{\mathrm{T}}$ (AV shunt, Corr) and $V_{\mathrm{T}}$ (IDIF, Uncorr) outcomes with both models. This was expected given the fairly gradual brain uptake of $\left[{ }^{11} \mathrm{C}\right] \mathrm{ITDM}$ and its slow wash-out profile similar to the findings reported in rats [8].

Finally, we explored whether a different approach simpler than $V_{\mathrm{T}}$ (IDIF, Uncorr) could be employed to measure $\left[{ }^{11} \mathrm{C}\right] \mathrm{ITDM}$ uptake. While SUV was not a reliable measurement, the use of SUVR resulted in more accurate values. Interestingly, SUVR (IDIF, Uncorr) $\left(r^{2}=0.907\right)$ performed almost as good as $V_{\mathrm{T}}$ (IDIF, Uncorr) $\left(r^{2}=0.954\right)$ when comparing both measurements to the $V_{\mathrm{T}}$ (AV shunt, Corr). While this might indicate that a static PET scan 60 to 90 min p.i. could be sufficient, it is important to underline that this approach might still need dynamic PET imaging in order to define the VOI for the IDIF following radioligand injection, unless a different strategy to extract a reliable IDIF is identified. Alternatively, it may be possible that a single blood sample collected during the PET scan could be sufficient to measure blood activity to derive the SUVR (Av shunt, Corr). Yet, such approach appeared less reliable $\left(r^{2}=0.692\right)$, invasive, and likely to be sensitive to noise due to the low activity in blood following decay of the radioligand at these later time points p.i.

\section{Conclusion}

We demonstrated that $\left[{ }^{11} \mathrm{C}\right] \mathrm{ITDM}$ selectively binds to mGluR1 in mice with no suitable reference region as shown both in vivo and in vitro. The use of IDIF and a scan duration of $90 \mathrm{~min}$ is recommended for accurate noninvasive estimates of $\left[{ }^{11} \mathrm{C}\right]$ ITDM binding. By applying the appropriate kinetic models, $\left[{ }^{11} \mathrm{C}\right]$ ITDM represents a promising tool for studying changes in mGluR1 during comparative studies of ageing and neurological disorders.

Acknowledgements. The authors thank Philippe Joye, Caroline Berghmans, and Annemie Van Eetveldt of the Molecular Imaging Center Antwerp (MICA) for their valuable assistance.

Funding Information. DB is supported by a $\mathrm{PhD}$ fellowship from the Research Foundation Flanders (FWO, 11W2516N/11W2518N). Antwerp 
University funded the work through a partial assistant professor position for JV and LW, and a full professor position for SStr and SSta. LW and SStr are also supported by Antwerp University Hospital through a departmental position.

Compliance with Ethical Standards. All applicable institutional and/or national guidelines for the care and use of animals were followed. Experiments were performed according to the European Committee Guidelines (decree 2010/63/CEE) and the Animal Welfare Act (7 USC 2131), and they were approved by the Ethical Committee for Animal Testing (ECD 2017-27) at the University of Antwerp (Belgium).

\section{Conflict of Interest}

The Antwerp University group received financial support from CHDI Foundation, Inc., a non-profit biomedical research organization exclusively dedicated to collaboratively developing therapeutics that will substantially improve the lives of HD-affected individuals. LM, CD, LL, MS, IMS are employees of CHDI Foundation, Inc. The authors declare no other potential conflicts of interest relevant to this work.

Open Access This article is distributed under the terms of the Creative Commons Attribution 4.0 International License (http:// creativecommons.org/licenses/by/4.0/), which permits unrestricted use, distribution, and reproduction in any medium, provided you give appropriate credit to the original author(s) and the source, provide a link to the Creative Commons license, and indicate if changes were made.

Publisher's Note. Springer Nature remains neutral with regard to jurisdictional claims in published maps and institutional affiliations.

\section{References}

1. Cartmell J, Schoepp DD (2000) Regulation of neurotransmitter release by metabotropic glutamate receptors. J Neurochem 75:889-907

2. Ribeiro FM, Paquet M, Cregan SP, Ferguson SS (2010) Group I metabotropic glutamate receptor signalling and its implication in neurological disease. CNS Neurol Disord Drug Targets 9:574-595

3. Abe T, Sugihara H, Nawa H, Shigemoto R, Mizuno N, Nakanishi S (1992) Molecular characterization of a novel metabotropic glutamate receptor mGluR5 coupled to inositol phosphate/Ca2+ signal transduction. J Biol Chem 267:13361-13368

4. Fotuhi M, Sharp AH, Glatt CE, Hwang PM, von Krosigk M, Snyder SH, Dawson TM (1993) Differential localization of phosphoinositidelinked metabotropic glutamate receptor (mGluR1) and the inositol 1,4,5-trisphosphate receptor in rat brain. J Neurosci 13:2001-2012

5. Pillai RL, Tipre DN (2016) Metabotropic glutamate receptor 5 - a promising target in drug development and neuroimaging. Eur J Nucl Med Mol Imaging 43:1151-1170

6. Li S, Huang Y (2014) In vivo imaging of the metabotropic glutamate receptor 1 (mGluR1) with positron emission tomography: recent advance and perspective. Curr Med Chem 21:113-123

7. Fujinaga M, Yamasaki T, Maeda J, Yui J, Xie L, Nagai Y, Nengaki N, Hatori A, Kumata K, Kawamura K, Zhang MR (2012) Development of N-[4-[6-(isopropylamino)pyrimidin-4-yl]-1,3-thiazol-2-yl]-Nmethyl-4-[11C]methylb enzamide for positron emission tomography imaging of metabotropic glutamate 1 receptor in monkey brain. J Med Chem 55:11042-11051

8. Yamasaki T, Fujinaga M, Yui J, Ikoma Y, Hatori A, Xie L, Wakizaka H, Kumata K, Nengaki N, Kawamura K, Zhang MR (2014)
Noninvasive quantification of metabotropic glutamate receptor type 1 with [(1)(1)C]ITDM: a small-animal PET study. J Cereb Blood Flow Metab 34:606-612

9. Yamasaki T, Maeda J, Fujinaga M et al (2014) PET brain kinetics studies of (11)C-ITMM and (11)C-ITDM, radioprobes for metabotropic glutamate receptor type 1 , in a nonhuman primate. Am J Nucl Med Mol Imaging 4:260-269

10. Kohara A, Takahashi M, Yatsugi S, Tamura S, Shitaka Y, Hayashibe S, Kawabata S, Okada M (2008) Neuroprotective effects of the selective type 1 metabotropic glutamate receptor antagonist YM202074 in rat stroke models. Brain Res 1191:168-179

11. Verhaeghe J, Bertoglio D, Kosten L, Thomae D, Verhoye M, van der Linden A, Wyffels L, Stroobants S, Wityak J, Dominguez C, Mrzljak L, Staelens S (2018) Noninvasive relative quantification of [(11)C]ABP688 PET imaging in mice versus an input function measured over an arteriovenous shunt. Front Neurol 9:516

12. Bertoglio D, Verhaeghe J, Kosten L, Thomae D, van der Linden A, Stroobants S, Wityak J, Dominguez C, Mrzljak L, Staelens S (2018) MR-based spatial normalization improves [18F]MNI-659 PET regional quantification and detectability of disease effect in the Q175 mouse model of Huntington's disease. PLoS One 13:e0206613

13. Bertoglio D, Verhaeghe J, Miranda A, et al. (2019) Validation and noninvasive kinetic modeling of [(11)C]UCB-J PET imaging in mice. J Cereb Blood Flow Metab:271678X19864081

14. Logan J, Fowler JS, Volkow ND, Wolf AP, Dewey SL, Schlyer DJ, MacGregor RR, Hitzemann R, Bendriem B, Gatley SJ, Christman DR (1990) Graphical analysis of reversible radioligand binding from timeactivity measurements applied to [N-11C-methyl]-(-)-cocaine PET studies in human subjects. J Cereb Blood Flow Metab 10:740-747

15. Lassen NA, Bartenstein PA, Lammertsma AA, Prevett MC, Turton DR, Luthra SK, Osman S, Bloomfield PM, Jones T, Patsalos PN, O'Connell MT, Duncan JS, Andersen JV (1995) Benzodiazepine receptor quantification in vivo in humans using [11C]flumazenil and PET: application of the steady-state principle. J Cereb Blood Flow Metab 15:152-165

16. Julien-Dolbec C, Tropres I, Montigon O, Reutenauer H, Ziegler A, Decorps M, Payen JF (2002) Regional response of cerebral blood volume to graded hypoxic hypoxia in rat brain. Br J Anaesth 89:287293

17. Paxinos G, Franklin K (2003) The mouse brain in stereotaxic coordinates. Academic Press; 2 edition (December 8, 2003)

18. Lammertsma AA, Bench CJ, Hume SP, Osman S, Gunn K, Brooks DJ, Frackowiak RSJ (1996) Comparison of methods for analysis of clinical [11C]raclopride studies. J Cereb Blood Flow Metab 16:42-52

19. Lehnert W, Gregoire MC, Reilhac A, Meikle SR (2012) Characterisation of partial volume effect and region-based correction in small animal positron emission tomography (PET) of the rat brain. Neuroimage 60:2144-2157

20. Yanamoto K, Konno F, Odawara C, Yamasaki T, Kawamura K, Hatori A, Yui J, Wakizaka H, Nengaki N, Takei M, Zhang MR (2010) Radiosynthesis and evaluation of [11C]YM-202074 as a PET ligand for imaging the metabotropic glutamate receptor type 1. Nucl Med Biol 37:615-624

21. Zanotti-Fregonara P, Chen K, Liow JS, Fujita M, Innis RB (2011) Image-derived input function for brain PET studies: many challenges and few opportunities. J Cereb Blood Flow Metab 31:1986-1998

22. Gill SS, Pulido OM, Mueller RW, McGuire PF (1999) Immunochemical localization of the metabotropic glutamate receptors in the rat heart. Brain Res Bull 48:143-146

Publisher's Note Springer Nature remains neutral with regard to jurisdictional claims in published maps and institutional affiliations. 